phys. stat. sol. (b) 63, 587 (1974)

Subject classification: 10.2 and $20.3 ; 19 ; 22.5 .2$

Institut de Physique de l'Université Neuchâtel, Switzerland

\title{
Luminescence and Optically Detected EPR of Close F-Center Pairs in KCl
}

\author{
By \\ P. A. SchnegG, C. JaCCARD, and M. Aegerter
}

By an appropriate optical treatment at RT the distance separating the members of $F$ center pairs can be decreased, giving rise to different optical and magnetic properties, revealed by the existence of a spin dependent non-radiative disexcitation mechanism. A model with an exchange spin-spin interaction is analyzed and compared with experiments at $\mathrm{LHe} \mathrm{T}$ (variation of the luminescence with an applied magnetic field, optical detection of the EPR, and relaxation processes). The observed effects are related with population changes in the ground state caused by optical pumping and they are sensitive to the mixing of the states produced by the different interactions. The average exchange energy is found to range between the hyperfine and the Zeeman energy depending of the state of aggregation. The paramagnetic resonance occurs in the ground state $(g=1.998$, linewidth $52 \mathrm{G})$ and the relaxation times range between 25 and $250 \mathrm{~ms}$, depending of the optical pumping rate. The relation predicted by the model between the luminescence variation and the relaxation rate agrees with the experiments.

En diminuant par un traitement optique adéquat fait à température ambiante la distance séparant les membres de paires de centres $\mathrm{F}$, on obtient des propriétés optiques et magnétiques différentes, révélées par l'existence d'un mécanisme de désexcitation non radiative dépendant des spins. Un modèle introduisant une interaction d'échange spin-spin est analysé et comparé à des expériences faites à basse température (variation de la luminescence en fonction d'un champ magnétique appliqué, détection optique de la RPE et processus de relaxation). Les effets observés sont liés aux changements des populations dans l'état fondamental causé par le pompage optique et ils sont sensibles au mélange des états de spin produits par les diverses interactions. On trouve une énergie d'échange moyenne située entre l'énergie hyperfine et celle de Zeeman, selon l'état d'agrégation. La résonance paramagnétique a lieu dans l'état fondamental ( $g=1,998$, largeur de raie $52 \mathrm{G}$ ) et les temps de relaxation se situent entre $\mathbf{2 5}$ et $\mathbf{2 5 0} \mathrm{ms}$ selon l'intensité du pompage optique. La relation prédite par le modèle entre la variation de la luminescence et le temps de relaxation est vérifiée par l'expérience.

\section{Introduction}

In alkali halide crystals containing a homogeneous concentration of about $10^{17} \mathrm{~F}$ centers $/ \mathrm{cm}^{3}$, an appreciable fraction of the centers may have a neighbor within a distance $R_{\mathrm{c}}$ which is small enough to allow an interaction. If one of the pair members is optically excited at low temperature with light in the $F$ band, it can return to the ground state by two ways: either a radiative transition (like an isolated center) or a non-radiative transition induced by the interaction [1]. The non-radiative disexcitation mechanism is still not well understood [2]. The interaction being partly magnetic, the non-radiative process can be influenced by static and microwave magnetic fields. This property has been 
used to detect optically the EPR of F centers in the ground and in the relaxed excited state with high sensitivity by means of the luminescence [3]. In the experiments presented here, the homogeneous center distribution is modified by successive optical bleachings of short duration in the $\mathrm{F}$ band at room temperature. Whereas the total concentration remains constant, the distances separating the pairs decrease, producing pairs referred below as "close pairs". Their magnetic and optical properties are completely different from the original ones [1], called below "distant pairs". We attribute this fact to the enhancement of the interaction near or above a significant level defined by the magnetic energy of the centers. In the following sections, we first describe the experimental procedure. Then the effect of the interaction on the pair states and energies is analysed and connected with the probability of the non-luminescent disexcitation. In Section 4 the dependence of the luminescence on an applied magnetic field is examined and in Section 5 a simplified model is given in order to calculate the kinetics of the population changes under transient optical and microwave excitations and it is compared with the experiments. The optical detection of the EPR and the spin relaxation are finally discussed.

\section{Experimental Procedure}

A KCl sample (K. Korth "Ultrapur") cleaved to the dimensions $6 \times 6 \times$ $\times 0.3 \mathrm{~mm}^{3}$ is placed on its thin side on the bottom of a cylindrical cavity working in the X-band (TE 111 mode). The cavity has three holes of $5 \mathrm{~mm}$ diameter to allow for $\mathrm{X}$-irradiation, optical bleaching and excitation, and luminescence detection (Fig. 1). The $\mathrm{F}$ centres are created by $\mathrm{X}$-irradiation at room temperature during $1 \mathrm{~h}(150 \mathrm{kV}, 10 \mathrm{~mA}$, filtered by $2 \mathrm{~mm} \mathrm{Al})$. In this way a homogeneous concentration of about $10^{17} \mathrm{~cm}^{-3}$ is achieved. Since the crystal is thin, the optical density ot the maximum of the F absorption band is of the order of 0.3 and the optical excitation is therefore homogeneous at all temperatures. The formation of "close pairs" is achieved by optical bleaching at room temperature: the light of a $250 \mathrm{~W}$ halogen lamp passed through two Schott filters (KG $33 \mathrm{~mm}$ and OG $5703 \mathrm{~mm}$ ) and different grey filters (OD from 0.15 to 2.6) is focussed on the crystal for short periods of time (usually $15 \mathrm{~s}$ ). In this way, the light flux can be varied from $10^{15}$ to $10^{17}$ photons $\mathrm{s}^{-1} \mathrm{~cm}^{-2}$, depending on the grey-filter configuration. Immediately after the bleaching, the temper-

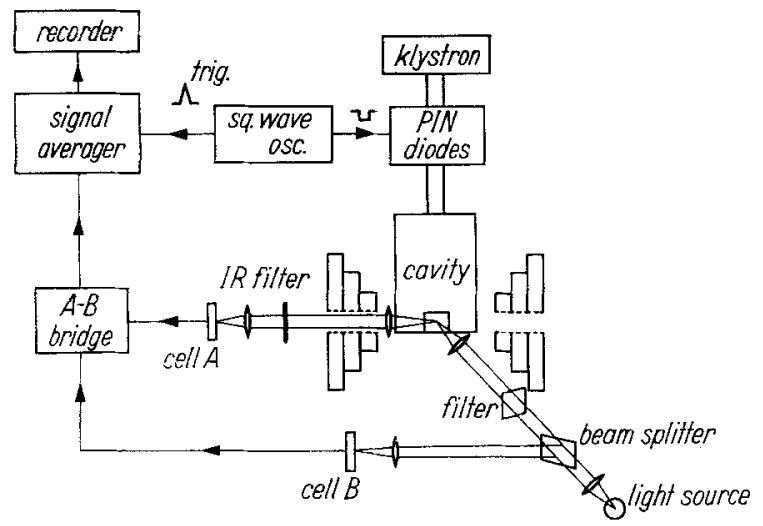

Fig. 1. Experimental arrangement 
ature is lowered to LHeT. The optical excitation is performed with the same arrangement, less the OG 570 filter. The luminescence is detected perpendicularly to the excitation beam through an IR filter (Wratten 87) by a PbS cell (Mullard $119 \mathrm{CPY}$ ). A fraction of the exciting light, equal to the luminescence intensity at zero magnetic field, is directed on a second cell by an adjustable beam splitter. The signal difference given by a bridge is either selectively amplified or sent to a signal averager (HP 5480). In order to study transient luminescent behavior, the excitation light can be chopped by a fast reed shutter ( 0 to $5 \mathrm{~Hz}$, rise time $10 \mathrm{~ms}$ ) and the microwaves can be modulated by a series of PIN diodes (HP 8735B). Relaxation times can be measured between $10 \mathrm{~ms}$ and several seconds. The LHeT cryostat (Andonian MHD-3L-30N) is placed between the poles of an electromagnet (Varian 3700).

\section{Pair States and Radiative Disexcitation}

In the case of "distant pairs", the two electron spins interact only with the homogeneous applied magnetic field $\boldsymbol{H}_{\mathbf{0}}$ and with the hyperfine field $\boldsymbol{H}_{\mathrm{N}}$ and $\boldsymbol{H}_{\mathrm{N}}^{*}$ produced by the neighboring nuclei. These nuclear fields are random and therefore differ at the two center sites, giving total fields $\boldsymbol{H}$ and $\boldsymbol{H}^{*}$. As it has been shown elsewhere [1] this difference, expressed by the angle $\psi$ between $\boldsymbol{H}$ and $\boldsymbol{H}^{*}$, produces a mixing of singlet $\left(\alpha \varphi_{\mathrm{s}}\right)$ and triplet $\left(\sigma \varphi_{\mathrm{a}}\right)$ spin states coupled with symmetrical and antisymmetrical orbital wave functions. The eigenstates are

$$
\begin{aligned}
& \Psi_{1}=\frac{1}{\sqrt{2}} \sin \left(\frac{\psi}{2}\right)\left(\varphi_{\mathrm{s}} \alpha+\varphi_{\mathrm{a}} \sigma_{0}\right)+\cos \left(\frac{\psi}{2}\right) \varphi_{\mathrm{a}} \sigma_{+1}, \\
& \Psi_{2}=\frac{1}{\sqrt{2}} \cos \left(\frac{\psi}{2}\right)\left(\varphi_{\mathrm{s}} \alpha+\varphi_{\mathrm{a}} \sigma_{0}\right)-\sin \left(\frac{\psi}{2}\right) \varphi_{\mathrm{a}} \sigma_{+1}, \\
& \Psi_{3}=\frac{1}{\sqrt{2}} \cos \left(\frac{\psi}{2}\right)\left(\varphi_{\mathrm{s}} \alpha-\varphi_{\mathrm{a}} \sigma_{0}\right)-\sin \left(\frac{\psi}{2}\right) \varphi_{\mathrm{a}} \sigma_{-1}, \\
& \Psi_{4}=\frac{1}{\sqrt{2}} \sin \left(\frac{\psi}{2}\right)\left(\varphi_{\mathrm{s}} \alpha-\varphi_{\mathrm{a}} \sigma_{0}\right)+\cos \left(\frac{\psi}{2}\right) \varphi_{\mathrm{a}} \sigma_{-1}
\end{aligned}
$$

with the energies

$$
\varepsilon_{1,4}= \pm \frac{1}{2}\left(g^{*} \beta H^{*}+g \beta H\right), \quad \varepsilon_{2,3}= \pm \frac{1}{2}\left(g^{*} \beta H^{*}-g \beta H\right) .
$$

The interaction between two centers which have been brought nearer to each other to form a "close pair" is assumed to be of the isotropic exchange type and it adds to the Hamiltonian a term $-J S_{1} \cdot S_{2}$. The eigenvalues of the energy in the perturbed system are related with the exchange coupling energy by the equation

$$
J=\frac{\left(\varepsilon^{\prime 2}-\varepsilon_{1}^{2}\right)\left(\varepsilon^{\prime 2}-\varepsilon_{2}^{2}\right)}{\varepsilon^{\prime}\left[\left(\varepsilon^{\prime 2}-\varepsilon_{1}^{2}\right) \cos ^{2}(\psi / 2)+\left(\varepsilon^{\prime 2}-\varepsilon_{2}^{2}\right) \sin ^{2}(\psi / 2)\right]} \quad \text { with } \quad \varepsilon^{\prime}=\varepsilon-\frac{J}{4}
$$


and the wave functions are mixed according to the following relations:

$$
\begin{aligned}
\widetilde{\Psi}_{j} & =\sum_{i} c_{j i} \Psi_{i}, \\
c_{j 1,4} & =D_{j}\left(\varepsilon_{j}^{\prime} \pm \varepsilon_{1}\right)\left(\varepsilon_{j}^{\prime 2}-\varepsilon_{2}^{2}\right) \sin \left(\frac{\psi}{2}\right) \\
c_{j 2,3} & =D_{j}\left(\varepsilon_{j}^{\prime} \pm \varepsilon_{2}\right)\left(\varepsilon_{j}^{\prime 2}-\varepsilon_{1}^{2}\right) \cos \left(\frac{\psi}{2}\right), \\
\frac{1}{2} D_{j}^{-2} & =\left(\varepsilon_{j}^{\prime 2}+\varepsilon_{1}^{2}\right)\left(\varepsilon_{j}^{2}-\varepsilon_{2}^{2}\right)^{2} \sin ^{2}\left(\frac{\psi}{2}\right)+\left(\varepsilon_{j}^{\prime 2}+\varepsilon_{2}^{2}\right)\left(\varepsilon_{j}^{\prime 2}-\varepsilon_{1}^{2}\right)^{2} \cos ^{2}\left(\frac{\psi}{2}\right) .
\end{aligned}
$$

Non-radiative disexcitation occurs either by the transient formation of a $F^{\prime}+$ vacancy pair (transfer of the excited electron to the neighboring $F$ center) or by phonon emission alone [2]. In both cases it requires a finite admixture of the spin antisymmetric state $\varphi_{\mathrm{s}} \alpha$ in the wave function. With

$$
\hat{\alpha}_{j}=\left\langle\varphi_{\mathrm{s}} \alpha \mid \widetilde{\Psi}_{j}\right\rangle=\sqrt{2} D_{j} \varepsilon_{j}^{\prime}\left[\left(\varepsilon_{j}^{\prime}-\varepsilon_{1}^{2}\right) \cos ^{2}\left(\frac{\psi}{2}\right)+\left(\varepsilon_{j}^{\prime 2}-\varepsilon_{2}^{2}\right) \sin ^{2}\left(\frac{\psi}{2}\right)\right]
$$

the non-radiative frequency is given by

$$
w_{\mathrm{t} j}=w_{\mathrm{t}_{\mathrm{o}}}(d)\left|\tilde{x}_{j}\right|
$$

where $\omega_{\mathrm{t}_{\mathrm{o}}}$ is the frequency for a pure $\varphi_{\mathrm{s}} \alpha$ state and depends on the pair separation $d$. If $v_{j}$ is the relative population of a state $j$, the luminescent probability for a pair is then

$$
P_{1}=\sum_{j} y_{j}\left(1+W_{\mathrm{t}}\left|\tilde{\alpha}_{i}\right|\right)^{-1} \quad \text { with } \quad W_{\mathrm{t}} \equiv \frac{w_{\mathrm{t}_{0}}}{w_{\mathbf{r}}},
$$

where $w_{\mathrm{r}}$ is the radiative disexcitation frequency. In the experimental conditions of this work $\left(T>2{ }^{\circ} \mathrm{K}\right.$ and $H_{0}<5 \mathrm{kG}$ ) the factors $v_{j}$ are all nearly equal to $1 / 4$ at equilibrium, i.e. without optical excitation.

\section{Luminescence as a Function of the Applied Magnetic Field}

According to this model, the luminescence depends on the magnetic field $\boldsymbol{H}_{0}$ through the angle $\psi$ and the energies $\varepsilon$ and $\varepsilon^{\prime}$. The overall relationship cannot be given analytically but it must be calculated by a Monte Carlo method used previously [1] in which the stochastic nuclear fields $\boldsymbol{H}_{\mathrm{N}}$ and $\boldsymbol{H}_{\mathbb{N}}^{*}$ are taken from a Gaussian distribution with a variance corresponding to the observed width of the EPR line. The energy levels, the antisymmetrical admixture, and the luminescent probability are then calculated for a given exchange energy $J$, The average luminescent probability gives the luminescent quantum efficiency for pairs only, normalized to unity. It has to be pointed out that in these experiments the total luminescence which is measured comes from the isolated centers and from the pairs and that there is no way to separate both components experimentally.

For "distant pairs" the model has been already confirmed by the observations [1]. Fig. 2 shows the variation of the luminescent quantum efficiency and of the 

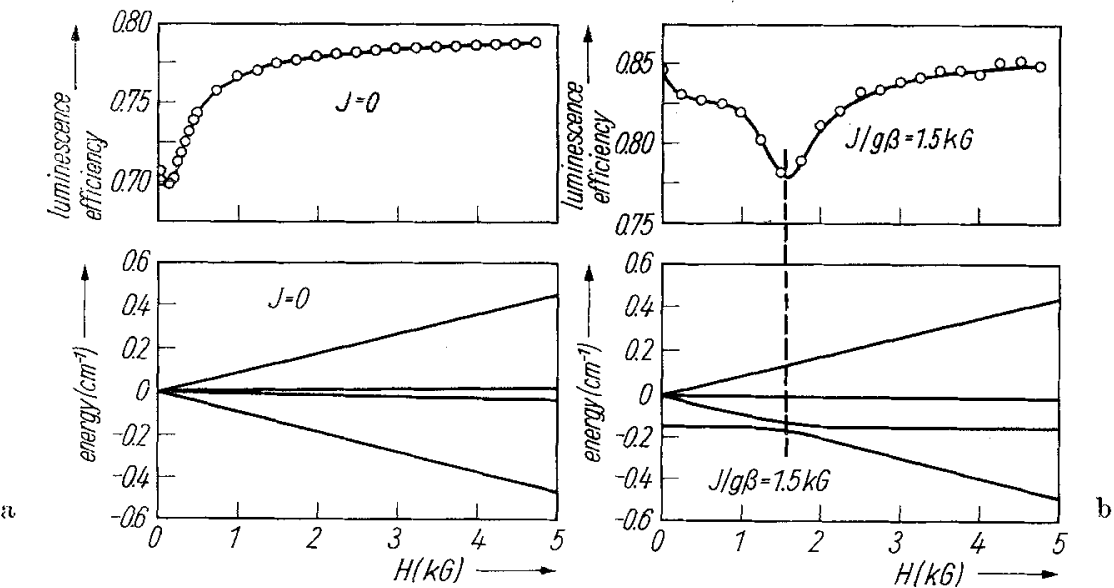

Fig. 2. Luminescence efficiency and energy levels of $F$ center pairs with $W_{t}=1$ as a function of the applied magnetic field, calculated for a $\mathrm{KCl}$ crystal. a) Without an exchange energy $(J=0) ; b)$ with an exchange energy $(J / g \beta=1.5 \mathrm{kG})$

energy levels as a function of the applied magnetic field calculated with the assumption that the crystal does not contain isolated centers but only "close pairs" with $W_{\mathfrak{t}}=1$. The left part of the figure shows the case of the "distant pairs" $(J=0)$ while in the right side the exchange energy is $J / g \beta=1.5 \mathrm{kG}$. The theoretical curve has a minumum near the field value corresponding to the exchange energy. Since such a dip has never been observed in the experiments, we have to assume a distribution of the $J$ 's resulting from a distribution of the pair separations. As a first approximation we choose an exponential distribution $p(J) \mathrm{d} J=\exp (-J / \bar{J}) \mathrm{d} J / \bar{J}$. The parameter $\bar{J}$, characterizing the different states of aggregation, is obtained by optimizing the fit of the calculated with the observed field variation of the luminescence, with a suitable scaling factor. Since the isolated centers do not respond to the field, their contribution is eliminated by this procedure. Such fits are shown in Fig. 3 (assuming $W_{t}=1$ ).

Fig. 3. Full curves represent the luminescent quantum yield of pairs of $\mathrm{F}$ centers in $\mathrm{KCl}$ (initial concentration $10^{17} \mathrm{~cm}^{-3}$ ) measured at $T=13.6^{\circ} \mathrm{K}$ after different optical treatments. The dots show the best fit with the theory assuming an exponential distribution of the exchange energy and $W_{\mathbf{t}}=1$. From (a) to (d), the value of $\bar{J} / g \beta$ is $0,342,1142$, and $\infty \mathrm{G}$, corresponding respectively to a quenched crystal and to RT bleaching of $5 \mathrm{~s}$ with grey filters ((b) $1.2 \times 10^{15}$, (c) $2.4 \times 10^{15}$, and (d) $1.2 \times 10^{16}$ photons $\mathrm{cm}^{-2}$ )

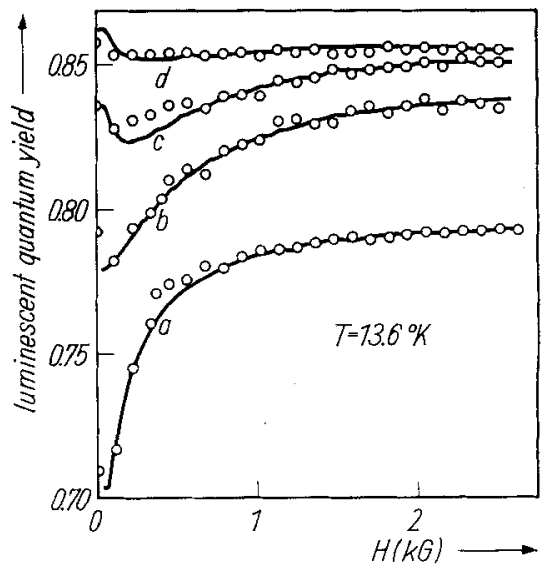




\section{Population Relaxation for "Close Pairs"}

Electronic transitions for "close pairs" between the different states are given in Fig. 4 a. The pumping rate $W_{\mathrm{p}}$ is proportional to the integrated light intensity absorbed in the $\mathrm{F}$ band; $w_{\mathbf{r}}$ and $w_{\mathrm{t}}$ are the radiative and non-radiative disexcitation rates. The rate coefficients $\left|\tilde{\alpha}_{j}\right|$ have been calculated with a distribution $p(J)$ corresponding to curve $\mathrm{c}$ of Fig. $3(J / g \beta=1142 \mathrm{G})$. The spin-lattice relaxation rate in the ground state is $W_{\mathbf{R}}$ and it can be increased by $W_{\mathrm{RT}}$ with resonant microwaves. Since the non-radiative disexcitation path may imply a delay in a transient state (e.g. $\mathrm{F}^{\prime}+$ vacancy) an intermediate level has been added with a transfer frequency $W_{\mathrm{B}}$ to the singlet ground state only. The net effect of the excitation light is then to populate the singlet level at the expense of the three other ones and therefore to decrease the luminescent quant um yield. Since the spin-spin relaxation rate $T_{2}^{-1}$ is large between the triplet states but very small between the triplet and the singlet, the system of Fig. 4 a can be approximated by the three species of Fig. $4 \mathrm{~b}$. The relaxed excited states can be left out because their population is quite small.

With $N_{\mathrm{t}}, N_{\mathrm{s}}$ are the triplet and singlet populations, $N$ is the total number of "close pairs" (in $\mathrm{em}^{-3}$ ) and

$$
W=W_{\mathrm{R}}+W_{\mathrm{RF}}, \quad k=\frac{w_{\mathrm{t}}}{\left(w_{\mathrm{t}}+w_{\mathrm{r}}\right)}, \quad w_{\mathrm{t}}=\sum_{j} \frac{w_{\mathrm{t} j}}{4}
$$

the rate equations for the populations are

$$
\begin{aligned}
& \dot{N}_{\mathrm{t}}=-\left(W+k W_{\mathrm{P}}\right) N_{\mathrm{t}}+3 W N_{\mathrm{s}}, \\
& \dot{N}_{\mathrm{s}}=\left(W-W_{\mathrm{B}}\right) N_{\mathrm{t}}-\left(3 W+W_{\mathrm{P}}+W_{\mathrm{B}}\right) N_{\mathrm{s}}+W_{\mathrm{B}} N .
\end{aligned}
$$

This system has the two relaxation times

$$
\tau_{\mathrm{f}}=\left(W_{\mathrm{P}}+W_{\mathrm{B}}\right)^{-1}, \quad \tau_{\mathrm{s}}=\left(4 W+k W_{\mathrm{P}}\right)^{-1}
$$

a
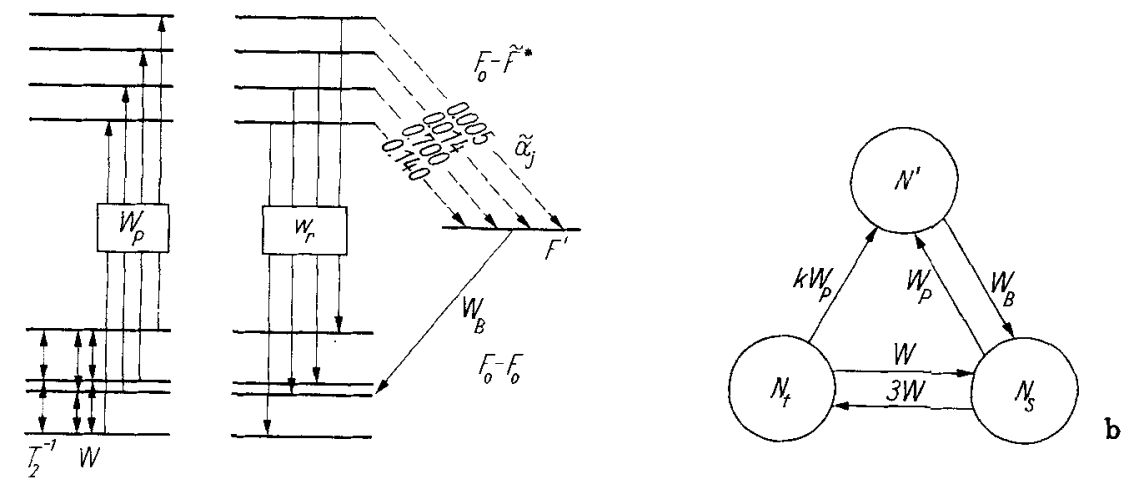

Fig. 4. a) Energy levels of a "close pair" with the different transition rates (see text).

b) Simplified model of a "close pair" 
The stationary values are

$$
\begin{aligned}
& N_{\mathrm{t}_{0}}=\frac{3 \beta^{\prime} N}{4 \beta^{\prime}+\alpha+k\left(3 \alpha+\alpha^{2}+\alpha \bar{\beta}^{\prime}\right)}, \\
& N_{\mathrm{s}_{\mathrm{o}}}=\frac{N_{\mathrm{t}_{\mathrm{o}}}(1+k \alpha)}{3},
\end{aligned}
$$

where $\alpha=W_{\mathrm{P}} / W$ and $\beta^{\prime}=W_{\mathrm{B}} / W$.

No time dependence can be detected in absorption measurements in the $\mathbf{F}^{\prime}$ band; this implies for this model a lifetime of the intermediate complexes $\left(F^{\prime}\right)$ shorter than $1 \mathrm{~ms}$, so that $1 / \beta^{\prime} \approx 0$. It is therefore not possible to distinguish between the two prevailing non-radiative disexcitation processes. $F^{\prime}$ formation cannot be excluded, since a large transfer rate to the ground state can depress the concentration variations below the detection level.

The transient behaviour of the luminescence, measured by chopping the excitation light (square wave modulation), is found to obey the law

$$
L(t)=L_{0}-\Delta L\left(1-\mathrm{e}^{-t / \tau}\right) .
$$

If $n$ is the total F-center number per unit volume and

$$
\Delta N_{\mathrm{t}_{\mathrm{o}}}=N_{\mathrm{t}_{\mathrm{o}}}(\alpha=0)-N_{\mathrm{t}_{0}}(\alpha \neq 0)
$$

is the population difference produced by the light, the formula above yields

$$
\begin{gathered}
\frac{L}{\Delta L}=\frac{n}{\Delta N_{\mathrm{t}_{\mathrm{v}}}(1-k)}=\frac{4 n}{3 N(1-k)}\left(1+\frac{4}{k \alpha}\right), \\
\tau=\tau_{\mathrm{s}}=\frac{1}{W(4+k \alpha)} .
\end{gathered}
$$

As it can be seen in Fig. 5, these laws, which are linear in $k \alpha$ or $(k \alpha)^{-1}$, agree qualitatively with the observed behavior at low light intensity. The relative luminescent variation is small ( 1 to $5 \%$ ) and the relaxation time varies between 25 and $250 \mathrm{~ms}$. A quantitative check is impossible because the constant $k$ is
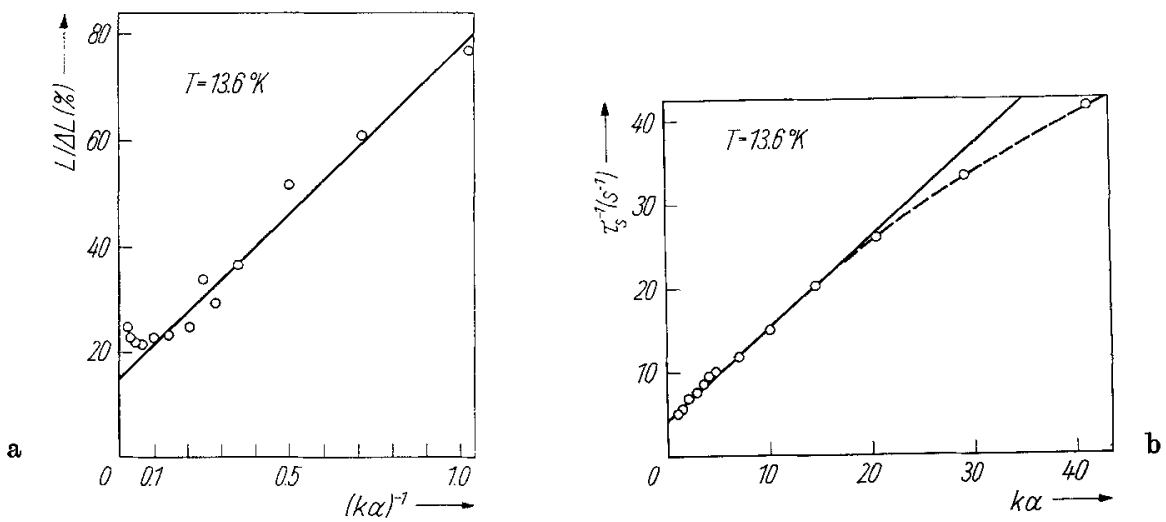

Fig. 5. a) Plot of the inverse of the relative luminescent variation due to a square optical excitation as a function of the inverse of the pumping light intensity $(k \alpha)^{-1}$. b) Plot of the relaxation rate $\tau_{\mathrm{s}}^{-1}$ as a function of the pumping light intensity $k \alpha . \tau_{\mathrm{s}}^{-1}=4 W+k W_{\mathrm{P}}$ 
not known a priori. However, the quantity $(L / \Delta L) \tau_{\mathrm{s}} W_{\mathrm{P}}$ should be independent of the pumping rate $W_{\mathbf{p}}$. The experimental results yield indeed a value which does not fluctuate more than $10 \%$ without any significant trend when the light intensity is changed by a factor of 20 , confirming the validity of the model.

Significant deviations are observed at high light intensity. They can be due to the ionization of the centers, thereby diminishing the number $N$. The effect on the relaxation time could be due to the inhomogeneity of the irradiation. At high intensity, the pairs near the surface become partly saturated and bleached. One observes then the pairs situated in the depth and since they receive a weaker light, their relaxation frequency is lower. From the ordinate at the origin of the extrapolated straight line of Fig. $5 \mathrm{a}$, it is possible to estimate the value of $k$. With $n=10^{17} \mathrm{~cm}^{-3}$ and $N / n$ about 0.1 , one finds $k=0.1$, indicating that the radiative disexcitation rate is one order of magnitude higher than the non-radiative one for "close pairs" in the triplet state.

\section{Optical Detection of Paramagnetic Resonance}

When the sample is subjected to a microwave field of a suitable frequency $\left(9.6 \mathrm{GHz}\right.$ for $\left.H_{0}=3400 \mathrm{G}\right)$, the "close pairs" show an increase of the luminescence of the same order as the quantity $\Delta L$ discussed in the preceding section. This is explained by transitions induced by the microwaves between the singlet and the triplet in the ground state, which equalize the populations. The luminescence is modified by EPR occurring in states in which the electron are weakly coupled with sharp energy levels, as it is the case in the excited state of "distant pairs" and in the ground state of "close pairs". But the resonance cannot be detected in the excited state of the latter because of the spread of the energy levels due to the wide exchange energy distribution. Since all the spin states are impure, the selection rule $\Delta S=0$ does not apply. The effect of the r.f. field is additive to the spin-lattice relaxation and with the relative intensity $\gamma=$ $=W_{\mathrm{RF}} / W_{\mathbf{R}}$ the resonance signal is described by

$$
\left(\frac{L}{\Delta L}\right)_{\gamma}-\left(\frac{L}{\Delta L}\right)_{\gamma=\infty}=A \gamma^{-1} ; \quad A=\frac{n(4+k \alpha)^{2}}{3 N(1-k) k \alpha} .
$$

This law is verified experimentally (Fig. 6), but only if all the pairs are excited simultaneously. This is not the case under ordinary conditions ( $H_{0}=$ const) because of the inhomogeneous broadening of the line by the nuclear fields. Only certain spins resonate and transmit their excitation to the other ones by spin

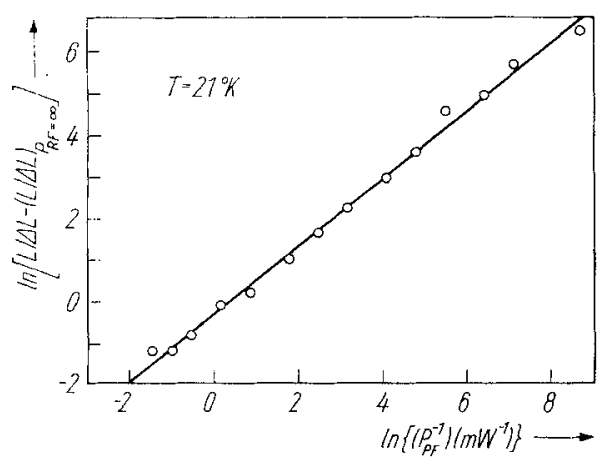

Fig. 6. Effect of the microwave power on the luminescence in the case of EPR of "close pairs" 
Fig. 7. Saturation of the relative luminescence variation observed as a function of the microwave power. (a) With magnetic field modulation of $30 \mathrm{G}$ around $H_{0}=3.4 \mathrm{kG}$; (b) with $H_{0}=3.4 \mathrm{kG}$ only

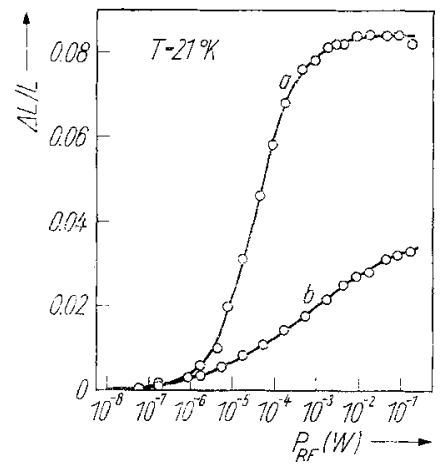

diffusion. This can be prevented by a $30 \mathrm{G}$ modulation of $H_{0}$ around the center of the resonance peak with a frequency larger than $30 \mathrm{kHz}$, which brings all the spins in resonance in a time which is very short with respect to the spinlattice relaxation time. With this method, saturation is produced with a r.f. power of $10 \mathrm{~mW}$ (Fig. $7 \mathrm{a}$ ) whereas with $170 \mathrm{~mW}$ without modulation only a third of the saturation value is reached (Fig. $7 \mathrm{~b}$ ).

The spin-lattice relaxation rate in the ground state can be measured by transient perturbation of the populations by chopping either the pumping light or the microwaves. Extrapolation to zero light intensity yields the value of $4 \mathrm{~W}$ (Fig. 5b). It is a function of temperature, as found by Feldman et al. [4] (Fig. 8), but also of the magnetic field (Fig. 9). At high field $\left(3400 \mathrm{G}\right.$ ) and $20^{\circ} \mathrm{K}$, the relaxation frequency is $4.5 \mathrm{~s}^{-1}$, slightly higher than for isolated centers for which it amounts to $3.5 \mathrm{~s}^{-1}$ [4]. For "close pairs", it is the sum of the single-center rate and of a modulation effect on the exchange energy by the phonons, with a strong dependence on the applied field. These properties will be disoussed in more details elsewhere.

The resonance of the "close pairs" is characterized by a nearly Gaussian line (Fig. 10a). The $g$-factor is decreasing with increasing temperature to an asymptotic value of $1.998 \pm 0.001$. This dependence is tentatively attributed to nuclear

Fig. 8. Dependence on temperature of the ground state spin-lattice relaxation rate $T_{1}^{-1}$ of "close pairs" at $H_{0}=3400 \mathrm{G}$ (extrapolated to $W_{\mathrm{P}}=0$ ) (•), compared with measurements on isolated centers by Feldman et al. [4] with electrolytically $(\Delta, \square)$ and additively (O) colored crystals

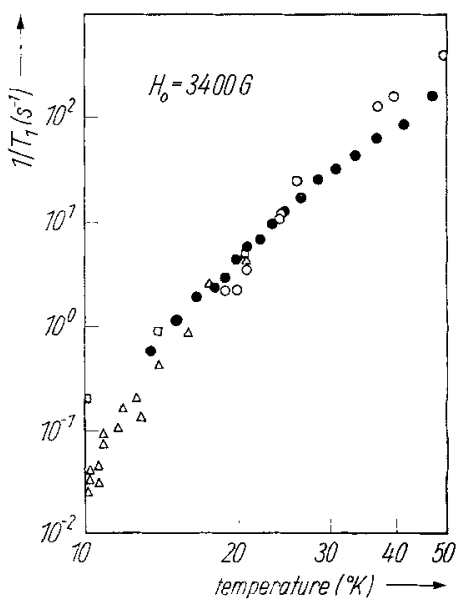




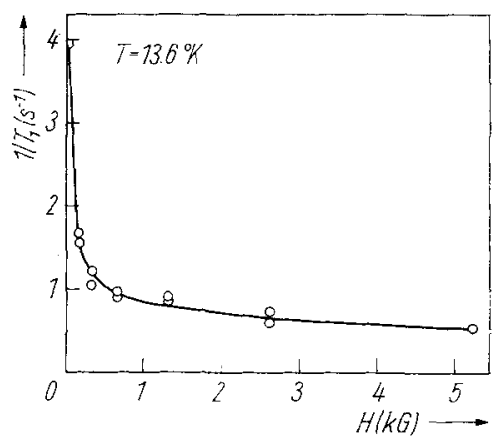

Fig. 9. Dependence on applied magnetic field of the ground state spin-lattice relaxation rate $T_{1}^{-1}$ of "close pairs" at $T=13.6^{\circ} \mathrm{K}$ extrapolated to

$$
W_{P}=0
$$

polarization. The FWHM of the line is $(52 \pm 1) \mathrm{G}$, a value improved with respect to previous measurements by a better signal-to-noise ratio. By increasing the bleaching at high temperature a second line is produced and then a third one (Fig. 10 b). ENDOR measurements with optical detection [5] confirm that they are due to transitions with $\Delta M= \pm 2$ or \pm 3 in pairs consisting of an F-center interacting with a neighboring $\mathbf{M}$ triplet or $\mathbf{R}$ quadruplet center, respectively.

If the crystal is cooled down very rapidly after bleaching, it is possible to keep some $\mathbf{F}$ center pairs with a separation shorter than the stability limit at room temperature. In the spectrum the central line splits in symmetrical pairs (Fig. $10 \mathrm{~b}, \mathrm{c}$ ). They can be attributed to the presence in the ground state of a significant interaction together with the predominance of certain pair separations. According to Schwörer and Wolf [6] who have calculated the exchange

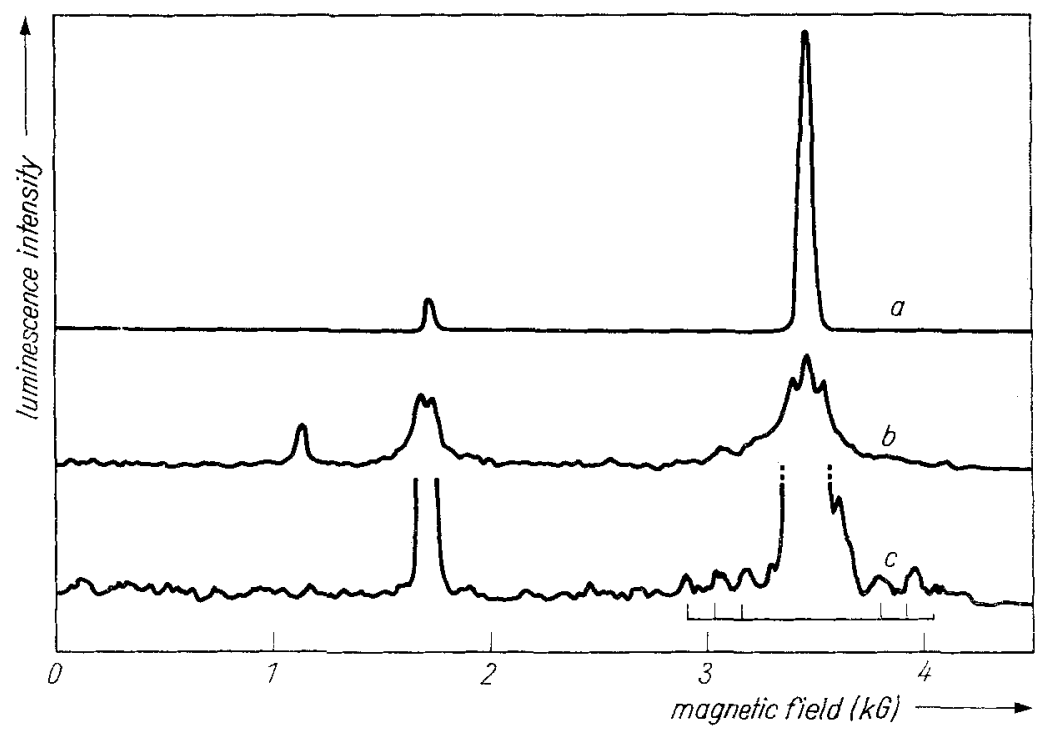

Fig. 10. Spectrum of the optically detected paramagnetic resonance for "close pairs". (a) Pairs F-F and F-M (triplet); (b) pairs F-F, F-M (triplet) and F-R (quartet) with a small effect of exchange in the ground state; (c) same as (a) but with a large effect of exchange in the ground state 
frequency as a function of the distance between two $F$ centers, a frequency of $10^{10} \mathrm{~s}^{-1}$ corresponding to the separation of the extreme peaks would indicate a distance of about four lattice constants between the members of the pair. It has not been possible to find an experimental treatment which produces pairs with a shorter distance. They should be unstable and coalesce spontaneously to form $M$ centers, as it can also be inferred from aggregation experiments [7].

\section{Conclusion}

$\mathrm{F}$ centers interact in $\mathrm{KCl}$ in two characteristic types of pairs which return to their ground state after $\mathrm{F}$ band excitation by a radiative or by a non-radiative process. The common property of both types is that the non-radiative disexcitation does not produce a sensitive number of $\mathrm{F}^{\prime}$ centers.

The "distant pairs" (separation between 30 and $80 \AA$ ) occur in quenched additively coloured crystals or in crystals $\mathrm{X}$-irradiated at room temperature and the response of their luminescence to magnetic and microwave fields is due to processes occurring in the relaxed excited state. They have been described at length in previous papers $[1,3]$. The "close pairs" are built up in bleached $\mathrm{X}$-rayed crystals, and their luminescence reflects population changes in their ground state. Their behavior is quite different from the "distant pairs" because of an exchange interaction which is significant mainly in the relaxed excited state. The interaction energy causes a dependence of the luminescence on static magnetic fields. The relaxation rate of the ground state populations, of the order of 1 to $100 \mathrm{~s}^{-1}$, is increased by the pumping light. It is influenced by the interaction, and it therefore depends of the static magnetic field and of temperature. The lifetime of any intermediate state on the non-radiative disexcitation path is shorter than $1 \mathrm{~ms}$. Since the optical pumping favors the non-luminescent singlet state, paramagnetic resonance is characterized by a sharp increase of the luminescence. If the pair separation is decreased, the exchange interaction becomes effective in the ground state too, and symmetrical pairs of new lines appear in the EPR spectrum.

These two types of $F$ center pairs constitute extreme cases, and they do not give quantitative information on the process of non-radiative disexcitation. Medium sized pairs have also been produced and they are related with significant changes of the $\mathrm{F}^{\prime}$ absorption (showing long lifetimes of 0.1 to $1 \mathrm{~s}$ ). They will be treated in a further paper dedicated to the disexcitation process.

\section{Acknowledgement}

The authors are indebted to the Swiss National Foundation for Scientific Research for the support of this work.

\section{References}

[1] C. Jaccard, Y. Ruedin, M. Aggerter, and P.-A. SchnegG, phys. stat. sol. (b) 50, 187 (1972).

[2] C. JACCARD and M. AEgERTER, Phys. Letters A 44, 391 (1973).

[3] Y. Ruedin and F. Porret, Helv. phys. Acta 41, 1294 (1968).

Y. Ruedin, P. A. SchNeGg, C. JACCARd, and M. AEgerTer, phys. stat. sol. (b) 54, 565 (1972); 55, 215 (1973).

[4] D. W. Feldman, R. W. Warken, and J. G. Castle, Jr., Phys. Rev. 13ə, A470 (1964). 
[5] P. A. Schneqa, C. JACCARd, and M. Aeqmiter, Phys. Letters A 42, 369 (1973); Proc. Europhysics Topical Conf. Lattice Defects in Ionic Crystals, Marseille-Luminy 1973, J. Physique 34, C 9-93 (1973).

[6] M. SCHWÖRER and H. C. WoLt, Z. Phys. 175, 457 (1963).

[7] C. H. SEAGER, D. O. WeLCH, and B. S. H. RoYCe, phys. stat. sol. (b) 49, 609 (1972); ธ0, 241 (1972).

(Received December 27, 1973) 